LA INTEGRACION DEL SISTEMA UNIVERSITARIO ESPAÑOL EN EL ESPACIO EUROPEO DE ENSEÑANZA SUPERIOR

Documento-Marco

MINISTERIO DE EDUCACIÓN, CULTURA Y DEPORTE

Febrero 2003 


\title{
LA INTEGRACION DEL SISTEMA UNIVERSITARIO ESPAÑOL EN EL ESPACIO EUROPEO DE ENSEÑANZA SUPERIOR
}

\author{
Documento-Marco
}

\section{INTRODUCCIÓN}

Entre los objetivos fundamentales de la Unión Europea se encuentra la coordinación de las políticas y normas legislativas de sus estados miembros en cuestiones relacionadas no sólo con el desarrollo económico, sino también con el progreso y el bienestar social de los ciudadanos. Este objetivo se ha extendido, en la última década, al ámbito de la educación y, muy singularmente, de la enseñanza superior, en el que diversos países, en un proceso que no hará sino incrementarse, han adoptado ya medidas conducentes a la reforma de la estructura y organización de sus enseñanzas universitarias para favorecer la construcción del Espacio Europeo de Educación Superior.

En este proceso han desempeñado un papel decisivo las redes de colaboración existentes entre las instituciones universitarias europeas, el desarrollo de programas de movilidad interuniversitaria, y el impulso generado por las declaraciones tanto de los responsables académicos de estas instituciones como por los ministros de educación de los países que conforman la Unión Europea (Sorbona, Bolonia y Praga). La declaración de La Sorbona (1998), en la que aparece por primera vez el concepto de Espacio Europeo de Educación Superior, pone de manifiesto una voluntad decidida de potenciar una Europa del conocimiento de acuerdo con las tendencias que predominan en los países más avanzados socialmente, en los que la extensión y calidad de la educación superior son factores decisivos en el incremento de la calidad de vida de los ciudadanos.

El Espacio Europeo de Enseñanza Superior significa un reto muy positivo para todos. Los estudios tendrán mayor transparencia y comparabilidad con beneficios para toda la sociedad y reportará a los estudiantes la organización de las enseñanzas en función de su aprendizaje. La introducción del crédito europeo como unidad del haber académico valora el volumen global de trabajo realizado por el alumno en sus estudios, no sólo las horas de clase. El diseño de los planes de estudio y las programaciones docentes se llevarían a cabo teniendo como eje del referencia el propio aprendizaje de los alumnos. El suplemento europeo al título ayudaría al reconocimiento más fácil y transparente por parte de otras universidades y organismos europeos de la formación adquirida. Y, en fin, la estructura de las enseñanzas que cursan y los niveles de los títulos que reciben al finalizar sus estudios serían más homogéneos con los correspondientes títulos y enseñanzas de los países de la Unión Europea favoreciendo su movilidad e integración en el mercado laboral.

Tan importante como el objetivo compartido de una armonización de los diversos sistemas que regulan las enseñanzas universitarias en cada estado miembro es la convicción, conjuntamente asumida, de que este proceso ha de llevarse a cabo con la máxima colaboración y participación de las propias instituciones de enseñanza superior así como con el máximo respeto a la diversidad de culturas y a la autonomía universitaria.

El Ministerio de Educación, Cultura y Deporte comparte plenamente estos objetivos y asume la responsabilidad que le compete de promover y llevar a cabo las modificaciones que hayan de realizarse en las estructuras de los estudios universitarios para alcanzar la plena integración del sistema español en el espacio europeo de enseñanza superior. Se trata de una previsión del Título XIII de la Ley Orgánica 6/2001, de 21 de diciembre, de Universidades. Por todo ello, remite al Consejo de 
Coordinación Universitaria, como máximo órgano consultivo y de coordinación del sistema universitario, y hace público el presente documento que contiene un conjunto de propuestas orientadas a servir de punto de partida para la reflexión que debe producirse en las universidades y administraciones educativas, y a posibilitar los acuerdos necesarios sobre los aspectos fundamentales del proceso de integración y que deberán orientar las normas jurídicas que se promulguen.

\section{LOS NUEVOS RETOS DEL SISTEMA UNIVERSITARIO ESPAÑOL}

Una adecuada comprensión de los cambios que han de introducirse en la estructura y en el funcionamiento de las enseñanzas universitarias sólo resulta posible concibiéndolos como eficaces instrumentos orientados al logro de una mejora de la calidad y de una plena adecuación de estas enseñanzas a las exigencias que una sociedad del conocimiento demanda. Es de justicia reconocer que las universidades españolas han experimentado, en los últimos años, profundas transformaciones entre las que, entre otras, merecen ser subrayadas las siguientes: un acelerado incremento del número de estudiantes que cursan estudios de educación superior, alcanzando una de las tasas más altas de escolarización universitaria de los países europeos; la creación de nuevas universidades y centros universitarios; el proceso de descentralización política y administrativa al haber asumido las Comunidades Autónomas sus competencias en el ámbito universitario y el propio ejercicio por parte de las Universidades del derecho constitucional de la autonomía universitaria; la ampliación y diversificación de la oferta educativa, tanto en titulaciones oficiales como en titulaciones propias; la potenciación y valoración de la actividad investigadora llevada a cabo en centros universitarios; el incremento de la movilidad internacional de profesores y estudiantes universitarios; $y$, en fin, el positivo desarrollo de los planes de evaluación y mejora de la calidad en el que han participado la mayor parte de las universidades.

Sin embargo, tal como se recoge en la exposición de motivos de la Ley Orgánica de Universidades, resulta necesaria una nueva ordenación de la actividad universitaria que permita a las Universidades "abordar, en el marco de la sociedad de la información y del conocimiento, los retos derivados de la innovación en las formas de generación y transmisión del conocimiento". La sociedad del conocimiento requiere innovaciones y cambios en las formas tradicionales de formación, producción, comunicación de la información y en el acceso a servicios públicos y privados. El bienestar de los ciudadanos, el dinamismo de la economía y la profundización en la participación democrática dependerán, en gran medida, de la forma en que las sociedades incorporen estos cambios y asuman las transformaciones sociales que conllevan.

En este contexto, la formación científica, humanística, artística y técnica adquiere una relevancia social fundamental no sólo como soporte del itinerario del aprendizaje para la actividad profesional, sino también como fundamento para el proceso de construcción de una comunidad europea de ciudadanos. La institución universitaria, que siempre ha jugado un papel decisivo en el desarrollo cultural, económico y social de los estados europeos, debe desempeñar una función decisiva en este nuevo escenario. En España, el previsible descenso de la presión demográfica en los próximos años comportará, sin duda, una excelente oportunidad para un mejor aprovechamiento de los recursos humanos y materiales, a la vez que resultará parcialmente compensada por el incremento de la demanda de todas las capas sociales de acceso a la educación superior y a la necesidad de atender a las exigencias de una formación continuada a lo largo de la vida. El desarrollo de la sociedad del conocimiento precisará de estructuras organizativas flexibles en la educación superior, que posibiliten tanto un amplio acceso social al conocimiento como una capacitación personal crítica que favorezca para la interpretación de la información y la generación del propio conocimiento. Se hace, 
pues, necesaria una nueva concepción de la formación académica, centrada en el aprendizaje del alumno, y una revalorización de la función docente del profesor universitario que incentive su motivación y que reconozca los esfuerzos encaminados a mejorar la calidad y la innovación educativa.

Por otro lado, el fenómeno de la globalización no se limita al ámbito económico sino que afecta también, de forma decisiva y positiva, a la transmisión de los conocimientos y a la formación superior. El carácter universal de la institución universitaria se verá aún más incrementado no sólo con la utilización de las nuevas tecnologías de la comunicación a distancia que eliminan barreras geográficas sino con una creciente movilidad de profesores, investigadores y alumnos que podrán ver atendidas sus demandas de educación universitaria de grado y postgrado en centros radicados en países distintos al que iniciaron su formación. Mantener este objetivo básico de la movilidad de los estudiantes universitarios de toda Europa significa construir más Europa, fomentando no sólo el acercamiento cultural y académico entre los alumnos, sino también encaminándolos hacia un mundo de ventajas laborales y profesionales. A este respecto, los estados miembros de la Unión Europea han adoptado la firme decisión de constituirse en centro de referencia mundial para la educación superior y las universidades españolas, en particular, están emplazadas a mantener o conseguir los niveles de calidad y competitividad que lo hagan posible.

Las propuestas contenidas en el presente documento-marco se orientan a la consecución de los objetivos mencionados en coherencia con lo manifestado en la exposición de motivos de la Ley Orgánica de Universidades: "Estos nuevos escenarios y desafíos requieren nuevas formas de abordarlos y el sistema universitario español está en su mejor momento histórico para responder a un reto de enorme trascendencia: articular la sociedad del conocimiento en nuestro país".

\section{EL ESPACIO EUROPEO DE EDUCACIÓN SUPERIOR}

La construcción del Espacio Europeo de Educación Superior es un proceso que se inicia con la Declaración de La Sorbona (1998) y que se consolida y amplía con la Declaración de Bolonia (1999), en las que los ministros europeos de educación instan a los estados miembros de la Unión Europea a desarrollar e implantar en sus países las siguientes actuaciones:

1. Adoptar un sistema de titulaciones comprensible y comparable para promover las oportunidades de trabajo y la competitividad internacional de los sistemas educativos superiores europeos mediante, entre otros mecanismos, de la introducción de un suplemento europeo al título.

2. Establecer un sistema de titulaciones basado en dos niveles principales. La titulación del primer nivel será pertinente para el mercado de trabajo europeo, ofreciendo un nivel de cualificación apropiado. El segundo nivel, que requerirá haber superado el primero, ha de conducir a titulaciones de postgrado, tipo master y/ o doctorado.

3. Establecer un sistema común de créditos para fomentar la comparabilidad de los estudios y promover la movilidad de los estudiantes y titulados.

4. Fomentar la movilidad con especial atención al acceso a los estudios de otras universidades europeas y a las diferentes oportunidades de formación y servicios relacionados.

5. Impulsar la cooperación europea para garantizar la calidad y para desarrollar unos criterios y unas metodologías educativas comparables.

6. Promover la dimensión europea de la educación superior y en particular, el desarrollo curricular, la cooperación institucional, esquemas de movilidad y programas integrados de estudios, de formación y de investigación. 
Posteriormente en el Comunicado de Praga (2001) se introducen algunas líneas adicionales:

1. El aprendizaje a lo largo de la vida como elemento esencial para alcanzar una mayor competitividad europea, para mejorar la cohesión social, la igualdad de oportunidades y la calidad de vida.

2. El rol activo de las universidades, de las instituciones de educación superior y de los estudiantes en el desarrollo del proceso de convergencia.

3. La promoción del atractivo del Espacio Europeo de Educación Superior mediante el desarrollo de sistemas de garantía de la calidad y de mecanismos de certificación y de acreditación.

La Cumbre de J efes de Estado celebrada en Barcelona en marzo de 2002 supuso un hito importante en el proceso de construcción del Espacio Europeo de Educación Superior: entre las Conclusiones de la Presidencia del Consejo Europeo figura expresamente la de crear las condiciones prácticas necesarias para garantizar la movilidad a todos los que participen en los ámbitos de la educación, la investigación y la innovación, así como reducir los obstáculos normativos y administrativos al reconocimiento profesional. Y aprobó un programa de trabajo que, entre otras acciones, solicita la introducción de instrumentos concebidos para garantizar la transparencia de los diplomas y cualificaciones (ECTS, suplementos a los diplomas y certificados, CV europeo) y una cooperación más estrecha en materia de diplomas universitarios en el marco del proceso La Sorbona-Bolonia-Praga. En fin, el Parlamento Europeo, en su informe de 24 de mayo de 2002 ha expresado su apoyo incondicional a la creación de este espacio educativo común, destacando su importancia y demandando el apoyo a las diferentes instituciones y países.

El compromiso establecido en la Declaración de Bolonia es alcanzar estos objetivos antes del final de la primera década del tercer milenio por lo que gran parte de los estados miembros y asociados de la Unión Europea han implantado, o tienen previstas, las reformas necesarias para la adaptación de sus sistemas nacionales de enseñanza superior. En la próxima reunión de ministros de educación a celebrar en Berlín en septiembre de 2003 se revisarán los progresos alcanzados en este ámbito e impulsar la coordinación de nuevas actuaciones para su consecución

\section{LA LOU Y EL ESPACIO EUROPEO DE EDUCACIÓN SUPERIOR}

La Ley Orgánica 6/2001, de 21 de diciembre, de Universidades en su Exposición de Motivos:

1) Establece como una de las finalidades del diseño de la nueva arquitectura normativa que reclama el sistema universitario español la de "integrarse competitivamente junto a los mejores centros de enseñanza superior en el nuevo espacio universitario europeo que se está comenzando a configurar".

2) Manifiesta que "la sociedad española necesita que su sistema universitario se encuentre en las mejores condiciones posibles de cara a su integración en el espacio europeo común de enseñanza superior".

3) Reconoce como uno de los objetivos esenciales de la Ley "impulsar la movilidad, tanto de estudiantes, como de profesores e investigadores dentro del sistema español pero también del europeo e internacional".

4) Declara que el estado ejercerá su responsabilidad de vertebración del sistema universitario mediante la financiación de programas orientados a dar cumplimiento a los objetivos previstos en la Ley entre los que incluye el de "promover la integración de las Universidades en el espacio europeo de enseñanza superior". 
Las previsiones que la LOU contempla para posibilitar las reformas que hayan de realizarse en las estructuras de los estudios están desarrolladas en el articulado correspondiente al título XIII de la propia ley Espacio Europeo de Enseñanza Superior:

1. Se adoptarán las medidas necesarias, en el ámbito de sus respectivas competencias, por parte del Gobierno, Comunidades Autónomas y Universidades para la plena integración del sistema español en el espacio europeo de enseñanza superior (Art. 87),

2. Se adoptarán las medidas para que los títulos oficiales expedidos por las Universidades españolas vayan acompañados del Suplemento Europeo al Título (Art. 88-1 y 3),

3. Se establecerán, reformarán o adaptarán las modalidades cíclicas de cada enseñanza y los títulos de carácter oficial y validez en todo el territorio nacional (Art. 88-2),

4. Se establecerán las medidas necesarias para adoptar el sistema europeo de créditos (Art. 88-3)

5. Se fomentará la movilidad de los estudiantes en el espacio europeo de enseñanza superior mediante programas de becas, ayudas y créditos al estudio (Art. 88-4).

A estas medidas deben agregarse aquellas otras contempladas en el artículo 88 para considerar habilitados, a los efectos previstos en la Ley, al profesorado de las Universidades de los Estados miembros de la Unión Europea, para la concurrencia a las pruebas de habilitación, concursos de acceso y convocatorias de contratos de profesorado por parte de los nacionales de esos Estados, y para favorecer la movilidad de profesores en el espacio europeo de enseñanza superior a través de programas y convenios específicos.

\section{PROPUESTAS PARA LA INTEGRACIÓN DEL SISTEMA UNIVERSITA- RIO ESPAÑOL EN EL ESPACIO EUROPEO DE EDUCACIÓN SUPERIOR}

La integración del sistema universitario español en el Espacio Europeo de Educación Superior requiere de propuestas concretas que desarrollen los distintos elementos conceptuales definidos en las declaraciones europeas y recogidos por la LOU. En especial, resultan decisivas las medidas que deban adoptarse sobre el sistema europeo de créditos, la estructura de las titulaciones, el Suplemento Europeo al Título (SET) y la garantía de la calidad. Asimismo, es necesario para que las propuestas sean viables y generen confianza en la comunidad universitaria, respañdar los programas que las promuevan y a los criterios y ritmos con que ha de llevarse a cabo la adaptación del sistema vigente de enseñanzas y títulos oficiales.

\subsection{LA IMPLANTACIÓN DEL SISTEMA DE CRÉDITOS EUROPEOS}

El crédito es la unidad de referencia sobre la que se estructuran y organizan los curricula formativos en la mayor parte de los países. En el actual sistema universitario español esta unidad se encuentra fundamentalmente definida en función de las horas de docencia, teórica o práctica, impartidas por los profesores. Ello implica diferencias en aspectos importantes con respecto al significado de la noción de crédito propugnada por la declaración de Bolonia y que está sirviendo de pieza básica en la armonización de las enseñanzas universitarias, por lo que se propone su sustitución por el denominado crédito europeo.

El sistema de créditos europeos, conocido como ECTS (European Credits Transfer System), nace y se desarrolla con los programas de movilidad de estudiantes para dar 
una respuesta a la necesidad de encontrar un sistema de equivalencias y de reconocimiento de los estudios cursados en otros países. La generalización de esta unidad de medida académica para todos los estudiantes es un objetivo fundamental para la creación del espacio europeo de educación superior, de forma que el trabajo desarrollado por un estudiante en cualquiera de las universidades de los estados miembros sea fácilmente reconocible en cuanto a nivel, calidad y relevancia.

El crédito europeo debe quedar definido como la unidad de valoración de la actividad académica en la que se integran las enseñanzas teóricas y prácticas, así como otras actividades académicas dirigidas y el volumen de trabajo que el estudiante debe realizar para alcanzar los objetivos educativos.

Su introducción en el sistema universitario español implica importantes diferencias con respecto al crédito vigente. Conviene subrayar, al respecto, que el crédito europeo no es una medida de duración temporal de las clases impartidas por el profesor, sino una unidad de valoración del volumen de trabajo total del alumno, expresado en horas, que incluye tanto las clases, teóricas o prácticas, como el esfuerzo dedicado al estudio y a la preparación y realización de exámenes. En resumen, esta nueva unidad de medida debe comportar un nuevo modelo educativo basado en el trabajo del estudiante y no en las horas de clase, o, dicho de otro modo, centrado en el aprendizaje de los estudiantes, no en la docencia de los profesores.

El sistema ECTS establece en 60 créditos el volumen de trabajo total de un estudiante a tiempo completo durante un curso académico. Por lo tanto, un semestre equivale a 30 créditos y un trimestre a 20 créditos. A título orientativo y considerando una actividad académica aproximada de 40 semanas/año y una carga de trabajo en torno a 40 horas/ semana, se establece para el crédito europeo un volumen de trabajo entre $25 \mathrm{y}$ 30 horas (1.500-1.800 horas de trabajo del estudiante/ año).

Las programaciones de cada una de las materias que conformen el plan de estudios de una titulación oficial deberán acogerse a esta unidad de medida integrando en la asignación de los créditos que a cada una le correspondan las clases docentes teóricas y prácticas, la preparación y realización de exámenes, horas de estudio que precisen, y los trabajos que los alumnos deban realizar para alcanzar los objetivos formativos de dichas materias. Asimismo, el reconocimiento de la labor docente de los profesores deberá incluir no sólo las horas dedicadas a impartir su docencia, sino también las dedicadas a organizar, orientar y supervisar el trabajo de los alumnos.

En este sentido, es propósito del Gobierno remitir para su informe por el Consejo de Coordinación Universitaria, en un plazo no superior a dos meses, el proyecto de normativa jurídica por el que se establezca el crédito europeo como unidad de medida del haber académico.

\subsection{LA ADAPTACIÓN DE LAS ENSEÑANZAS Y TÍTULOS OFICIALES UNIVERSITARIOS}

La propuesta que se somete a consideración de las universidades y administraciones educativas de las Comunidades Autónomas es la de armonizar la estructura cíclica de las enseñanzas universitarias con el esquema propugnado por la Declaración de Bolonia y que está vigente en gran parte de los Estados de la Unión Europea y extracomunitarios: Un primer nivel de grado que dará lugar a la obtención de un título con cualificación profesional en el mercado laboral europeo, y un segundo nivel de postgrado, para cuyo acceso será necesario haber superado el primero, y que podrá dar lugar a la obtención del Título de Master y/ o Doctorado. 
El Gobierno, previo informe del Consejo de Coordinación Universitaria, establecerá la modalidad cíclica de cada enseñanza y los títulos oficiales que les correspondan, respetando, en todo caso, las directivas europeas vigentes para una determinada titulación académica o profesional.

\subsubsection{El primer nivel: el grado}

Los objetivos formativos de las enseñanzas oficiales de nivel de grado tendrán, con carácter general, una orientación profesional, es decir, deberán proporcionar una formación universitaria en la que se integren armónicamente las competencias genéricas básicas, las competencias transversales relacionadas con la formación integral de las personas y las competencias más específicas que posibiliten una orientación profesional que permita a los titulados una integración en el mercado de trabajo. A este respecto, resultará esencial en el proceso de diseño y elaboración de las enseñanzas oficiales del nivel de Grado no sólo su armonización con las titulaciones consolidadas en otros países europeos en cada uno de los ámbitos científicos, técnicos y artísticos, sino la estrecha colaboración entre los responsables académicos y los de las asociaciones y Colegios Profesionales.

Se somete a consideración del Consejo de Coordinación Universitaria las siguientes propuestas alternativas:

a) El primer nivel dará lugar al título oficial de Licenciado, Ingeniero o Arquitecto. Su obtención requerirá haber obtenido 240 créditos europeos.

b) El primer nivel dará lugar al título de Licenciado, Ingeniero o Arquitecto. Su obtención requerirá completar entre 180 y 240 créditos europeos en las enseñanzas correspondientes.

Estas titulaciones deberán diseñarse en función de unos perfiles profesionales con perspectiva nacional y europea y de unos objetivos que deben hacer mención expresa de las competencias genéricas, transversales y específicas (conocimientos, capacidades, y habilidades) que pretenden alcanzarse.

Los requisitos para la obtención de los títulos universitarios oficiales de grado, y las directrices generales de los planes de estudios, serán establecidos por el Gobierno, bien por su propia iniciativa, previo informe del Consejo de Coordinación Universitaria, o a propuesta de este Consejo.

Asimismo, deberá ser posible una cierta flexibilidad que permita a las universidades diversificar su oferta, intensificando o personalizando alguna de las competencias específicas relacionadas con la orientación profesional, así como establecer itinerarios de libre configuración curricular. Pero, en ningún caso, estos itinerarios podrán ser reconocidos como especialidades ni reflejados en el título oficial de Grado.

Tras la aprobación por el Gobierno de las directrices generales de cada titulación, las universidades elaborarán los planes de estudio y, previo informe favorable de la Comunidad Autónoma correspondiente, los remitirán al Consejo de Coordinación Universitaria para su homologación. Una vez homologado el plan de estudios, el Gobierno homologará los títulos correspondientes a los efectos de que la Comunidad Autónoma pueda autorizar la impartición de las enseñanzas y la Universidad proceder, en su momento, a la expedición de los títulos.

Asociado con los perfiles profesionales, deberá definirse un catálogo de títulos de primer nivel, tomando como punto de partida el actual, pero propiciando una disminución mediante las fusiones o agrupaciones necesarias para racionalizar el conjunto tanto desde el punto de vista nacional como europeo. 
Para que esta compleja y relevante tarea arranque con unos principios básicos comunes a todas las titulaciones, el Gobierno elaborará una norma jurídica de carácter general que defina y regule las nuevas modalidades cíclicas de las enseñanzas oficiales. El proyecto correspondiente será remitido al Consejo de Coordinación Universitaria para su informe en un plazo no superior a dos meses.

\subsubsection{El segundo nivel: el postgrado}

De acuerdo con lo establecido en la Declaración de Bolonia, el segundo nivel de las enseñanzas universitarias, para cuyo acceso se requerirá haber superado el primero, conducirá a la obtención de los títulos de Master y/ o Doctor.

En el diseño de la estructura de este nivel existen diversas opciones que están vigentes en otros países. Todas ellas cuentan con ventajas e inconvenientes. Con carácter general, puede resultar conveniente establecer que la estructura Master-Doctorado tenga un carácter secuencial de modo que, para el conjunto de las titulaciones, el acceso al período de elaboración de la tesis doctoral sólo resulte posible tras obtener el título de Master en un programa de postgrado. En todo caso, esta exigencia sería necesaria en los Master no orientados hacia la investigación, y estrictamente profesionales. Pero también puede entenderse que esta estructura es demasiado rígida y que, en consecuencia, debería abrirse la posibilidad de que, además de lo anterior, puedan diseñarse Programas de Postgrado en los que los alumnos, tras haber cursado un número significativo de créditos, puedan ser admitidos por un Departamento o Instituto Universitario de Investigación para iniciar en ellos la elaboración de su trabajo de investigación doctoral.

Por ello, se somete a consideración del Consejo de Coordinación Universitaria:

a) si la obtención del título de Master debe ser un requisito para acceder en cualquier caso al doctorado,

b) si esta exigencia debe, como requisito previo, debe ser la habitual pero abriendo la posibilidad de que se regulen también las circunstancias extraordinaria que permitan diseñar programas de postgrado en los que se establezcan los requisitos para el acceso, por ejemplo, haber completado un número de créditos de ese programa, pero sin necesidad de haberlo completado y haber obtenido el Título de Master,

c) o, en fin, si esta posibilidad no debe considerarse con carácter extraordinario sino generalizable a determinados tipos de Master en función de sus objetivos y contenidos formativos. En cualquiera de los tres casos, el Gobierno establecerá, previo informe del Consejo de Coordinación Universitaria, los requisitos generales de acceso a los estudios de Postgrado y al Doctorado.

Las Universidades, tras la elaboración y aprobación de los Programas de Postgrado en la forma en que determinen sus Estatutos, deberán solicitar el informe de la Comunidad Autónoma competente y remitirlos al Consejo de Coordinación Universitaria para su homologación. El desarrollo efectivo de las enseñanzas de estos Programas de Postgrado conducentes a la obtención de títulos oficiales, serán sometidos a evaluación de la Agencia Nacional de Evaluación de la Calidad y Acreditación, de acuerdo con lo previsto en el artículo 35 de la Ley Orgánica de Universidades.

\section{El título oficial de Master}

La elección de la denominación de "Master" para los títulos de postgrado tiene indudables ventajas por ser el más generalmente utilizado en otros sistemas universitarios de la Unión Europea y países extracomunitarios. Denominaciones alternativas serían de la "Magister" o Master Universitario". 
Los objetivos formativos serán más específicos que los de Grado y deberán estar orientados hacia una mayor profundización intelectual, posibilitando un desarrollo académico disciplinar e interdisciplinar, de especialización científica, de orientación a la investigación o de formación profesional avanzada.

La obtención del título de Master requerirá haber completado entre un mínimo de 60 y un máximo de 120 créditos europeos, en función de la formación previa acreditada y de la orientación investigadora, científica o profesional que tengan cada uno de estos estudios de postgrado.

Los programas deberán tener una estructura flexible y un sistema de reconocimiento y de conversión que permitan el acceso desde distintas formaciones previas. Los contenidos de estas titulaciones deberán definirse en función de las competencias científicas y profesionales que hayan de adquirirse.

Dada la diversidad y carácter especializado de estos estudios de Postgrado y a fin de favorecer la flexibilidad que han de gozar para adaptarse a los cambios que en su ámbito de conocimientos científico-tecnológicos y competencias, el Gobierno regulará los requisitos generales de estos estudios, pero no establecerá directrices generales sobre sus contenidos.

La existencia de Masters oficiales será compatible con la de Masters como titulaciones propias no oficiales impartidas por las universidades u otros centros de formación superior. Esta última circunstancia deberá hacerse constar en los diplomas o certificados que aquéllas y éstos expidan.

La obtención del Título del Master exigirá haber completado la totalidad de los créditos y haber superado una prueba conjunta de evaluación o trabajo final del Master defendido ante un Tribunal. Esta prueba comportará una calificación diferenciada de los méritos de los estudiantes aprobados.

Los Títulos oficiales de Master serán expedidos por el Rector de la Universidad y en ellos se hará constar la expresión "Master en.... por la Universidad de..."

A diferencia de los Títulos de Grado, cabe considerar la conveniencia de que las enseñanzas conducentes a la obtención de estos títulos de Postgrado puedan ser programadas incorporando itinerarios curriculares distintos y especialidades dentro del correspondiente ámbito científico, tecnológico o profesional. Esta posibilidad tendría la ventaja de evitar la fragmentación de los contenidos en titulaciones distintas, reduciendo el número de denominaciones de Master oficiales a la vez que introduciría una cierta flexibilidad para que puedan adaptarse a nuevas exigencias de formación especializada. En tal caso, la denominación de estas especialidades podría ser añadida en los Títulos tras la denominación del Master: "Master en por la Universidad de ........".

Especialidad en

\section{El título de Doctor}

Entre los objetivos fundamentales de la reforma propuesta se encuentra la revalorización de los estudios de doctorado y la mejora de los niveles de excelencia en el grado superior académico. El futuro del sistema universitario español pasa, en gran medida, por un incremento de los niveles de competitividad de estos estudios, que los hagan atractivos a nivel internacional, así como por un mayor reconocimiento de la formación que proporcionan por parte de empresas e instituciones no académicas. 
Esta etapa de la formación consistirá en la elaboración y defensa de una tesis doctoral que deberá contener resultados originales de investigación.

Los estudiantes podrán solicitar su inscripción en un Programa de Doctorado ante el Departamento o Instituto Universitario de Investigación responsable del mismo, que establecerá los requisitos de admisión y la formación previa requerida. La inscripción oficial de los estudiantes de Doctorado en la Universidad conllevará el disfrute de todos sus derechos como estudiantes universitarios.

La Comisión responsable en cada universidad de aprobar y coordinar los estudios de Doctorado, con anterioridad a proceder a la autorización o no de la defensa de la tesis, someterá la misma a examen de dos revisores externos que habrán de ser doctores de reconocido prestigio en la especialidad o materia sobre la que verse la tesis, ajenos a la universidad en que haya de defenderse.

Los títulos de Doctor expedidos por las Universidades incluirá la mención “Doctor por la Universidad...." seguida de la referencia a la universidad que corresponda.

El Gobierno podrá establecer una mención de calidad a los títulos de Doctor que cumplan con los requisitos que oportunamente se establezcan.

El desarrollo de todos estos puntos clave que conformarán los estudios universitarios de Postgrado en España será incluido en un proyecto de Real Decreto que el Gobierno enviará para informe del Consejo de Coordinación Universitaria, en un plazo no superior a dos meses.

\subsection{EL SUPLEMENTO EUROPEO AL TÍTULO: UN ELEMENTO DE TRANSPARENCIA}

El Suplemento Europeo al Título es una iniciativa europea auspiciada por el Consejo de Europa, la UNESCO y la Asociación Europea de Universidades. Constituye un elemento de transparencia ya que su objetivo fundamental es hacer comprensibles y comparables los títulos universitarios en Europa por medio de una información académica y profesional relevante para la sociedad, la universidad y los empleadores.

El Suplemento Europeo al Título es un modelo de información unificado, personalizado para el titulado universitario, sobre los estudios cursados, su contexto nacional y las competencias y capacidades profesionales adquiridas. Pretende ser un documento fácilmente comprensible, abierto para incorporar el aprendizaje a lo largo de la vida, acreditando los conocimientos adquiridos por cada persona en diferentes instituciones europeas de educación superior.

Para la implantación del Suplemento Europeo al Título en España se plantean dos etapas. Una primera fase transitoria, hasta que se implanten las nuevas titulaciones estructuradas conforme a los créditos europeos, en la que se podrá expedir el Suplemento para las titulaciones actuales, y una segunda fase definitiva, una vez implantadas las nuevas titulaciones conforme al sistema de créditos europeo.

La expedición del Suplemento Europeo al Título es una responsabilidad de las universidades aunque el Consejo de Coordinación Universitaria deberá pronunciarse sobre algunos de sus epígrafes como los principales campos de estudio de las diferentes titulaciones, la condición profesional de cada título oficial y la información sobre el sistema nacional de enseñanza superior. 


\subsection{ACREDITACIÓN ACADÉMICA Y CALIDAD}

La mejora de la calidad del sistema universitario es una pieza clave sobre la que pivota la construcción del Espacio Europeo de Educación Superior, como lo prueba el hecho de que, desde el principio, la calidad sea una referencia en todos los textos y así se enfatizara en el comunicado de los ministros educación europeos tras la reunión de Praga de 2001. La consecución del objetivo de lograr que Europa se convierta en lugar de referencia en el ámbito de la formación universitaria para estudiantes de todos los países, pasa necesariamente por una garantía de la calidad fundamentada en mecanismos y procesos de evaluación, certificación y acreditación.

La Ley Orgánica de Universidades reconoce como uno de sus objetivos básicos la mejora de la calidad del sistema universitario en su conjunto y en todas y cada una de sus vertientes. A tal fin crea la Agencia Nacional de Evaluación de la Calidad y Acreditación que, junto con los órganos de evaluación que puedan crearse en las Comunidades Autónomas, serán las responsables, en sus respectivos ámbitos de competencias, de llevar a cabo las políticas previstas de evaluación, certificación y acreditación, si bien la Agencia Nacional de Evaluación de la Calidad y Acreditación será la que intervenga en la elaboración de informes conducentes a la homologación por el Gobierno de títulos de carácter oficial y validez en todo el territorio nacional.

La mutua confianza entre las instituciones de enseñanza superior y el reconocimiento de las titulaciones que éstas expidan debe tener como soporte básico una metodología común y contrastada de evaluación de la calidad. La movilidad y el reconocimiento de estudios no sólo requieren un clima de confianza y de transparencia sino también una correspondencia entre los elementos básicos de la formación en los distintos sistemas de educación superior. Los sistemas de garantía de la calidad jugarán un papel importante para conseguir unos elevados niveles de excelencia que facilitarán la comparabilidad y el reconocimiento de las calificaciones y títulos en toda Europa. Pero para ello será también necesaria una convergencia en los sistemas de garantía de calidad de los distintos estados.

La definición de criterios y estándares mínimos compartidos por las agencias responsables de asegurar la calidad y algunos criterios comunes sobre sistemas de acreditación y evaluación de estudios e instituciones serán elementos necesarios en la construcción del Espacio Europeo de Educación Superior. A tal fin, deberá desarrollarse al máximo la colaboración entre la Agencia Nacional de Evaluación de la Calidad y Acreditación, y las agencias de otros países de la Unión Europea en el marco de la acreditación de títulos oficiales en los respectivos países.

\section{LA CORRESPONSABILIDAD INSTITUCIONAL EN LA PUESTA EN MARCHA DEL PROCESO}

Nadie puede ni debe sentirse ajeno al impulso que implicará, en nuestro sistema educativo, su incorporación al Espacio Europeo de Educación Superior. Las administraciones, todos los sectores de las universidades, y el conjunto de la sociedad deben incrementar su respaldo y exigencias, al mismo tiempo que las universidades tendrían que continuar incrementando la eficiencia y transparencia de sus gastos. Un modelo de educación superior más eficiente, más competitivo y de mayor calidad requiere, en algunos sectores del sistema, inversiones e incentivos que ayuden a conseguir un resultado óptimo del esfuerzo que la comunidad universitaria debe realizar adecuando sus estructuras actuales, modificando los procedimientos de aprendizaje e incentivando a las personas e instituciones involucradas. 
Las universidades públicas españolas cuentan con unas actividades financiadas desde el gobierno central, otras, las más cuantiosas, financiadas desde las comunidades autónomas y, por último, otras financiadas desde los recursos captados o generados por las propias universidades. Por ello, la financiación necesaria para el proceso de convergencia debe provenir de los distintos agentes mencionados en función de sus responsabilidades legales. En cualquier caso, la financiación pública, afectará a los dos niveles, grado y postgrado, de la educación superior.

La integración efectiva de las enseñanzas oficiales del sistema universitario español en el marco del espacio europeo exigirá no sólo la financiación de programas específicos de movilidad de profesores y alumnos, tal como se establece en los artículos 88.4 y 89.4 de la Ley Orgánica de Universidades, sino también la financiación destinada a posibilitar la plena integración de las titulaciones en ese espacio. Esta exigencia debe ser atendida, conjuntamente, por el Gobierno, las Comunidades Autónomas y las propias Universidades.

Los costes derivados del proceso de convergencia tendrán que distribuirse a lo largo de dos periodos: el de promoción e incentivación del proceso, para su eficaz y rápida puesta en marcha, y el de su efectiva y generalizada implantación. Tanto en un período como en el otro, cada agente tendrá que evaluar y decidir la magnitud de su participación, si bien la idea de un consenso amplio en medios y objetivos debería ser considerada por todos ellos a fin de lograr el máximo rendimiento de las inversiones y los mejores resultados. A tal fin, en la primera etapa, el Gobierno se compromete a financiar estudios sobre las implicaciones de este proceso en el sistema universitario español y promover encuentros y grupos de trabajo interuniversitarios sobre aspectos específicos de la reforma en titulaciones oficiales.

Esta situación ofrece, por lo demás, una fecunda oportunidad para diseñar políticas coordinadas y para ensayar modelos de prueba y error a partir de los datos que se vayan obteniendo de la diferentes experiencias que se están desarrollando y de las que se desarrollen en el futuro. Este aspecto es especialmente relevante para todo aquello relacionado con la nueva estructura de las titulaciones, el modelo de formación centrado en el aprendizaje de los estudiantes y la aplicación de los créditos europeos.

\section{LA TRANSICIÓN DE LAS TITULACIONES ACTUALES AL NUEVO SISTEMA DE LAS ENSEÑANZAS UNIVERSITARIAS}

Resultan altamente recomendables las experiencias-piloto, de carácter interuniversitario, que puedan llevarse a cabo en el ámbito de titulaciones específicas y de las cuales puedan extraerse consecuencias a tomar en cuenta en el diseño que finalmente deban adoptar. Asimismo, es propósito del Gobierno que la implantación de estas nuevas enseñanzas no se lleve a cabo homogéneamente en un mismo curso académico sino que el ritmo de esta adaptación se adecue a los acuerdos que el Consejo de Coordinación Universitaria adopte sobre cada titulación y a las decisiones de las propias Universidades.

La complejidad y la relevancia de los cambios que comportará la integración del sistema universitario español hace recomendable que el proceso de transición al nuevo sistema esté presidido por los siguientes criterios:

a) Flexibilidad: tanto en la reforma de las titulaciones según los ámbitos científicos y profesionales como en el calendario que las propias universidades establezcan para el ritmo de implantación en ellas de las nuevas enseñanzas. 
b) Simplificación: tanto en los procesos de adaptación de los alumnos como en los periodos de convivencia de ambos sistemas.

Por ello, se propone que las actuales titulaciones de Diplomado, Ingeniero Técnico, Arquitecto Técnico, Licenciado, Ingeniero y Arquitecto, así como los estudios conducentes al Diploma de Estudios Avanzados (DEA) de los actuales programas de doctorado, puedan continuar impartiéndose, en paralelo con las nuevas titulaciones, durante un período transitorio hasta que se generalice el proceso de adaptación. En cualquier caso la fecha de extinción de estos planes no deberá superar el 2010.

Las titulaciones actuales se extinguirán al finalizar dicho plazo transitorio, sin posibilidad de prórroga. La implantación de un nuevo título en una universidad supondrá el inicio del proceso de extinción simultánea del antiguo si corresponde a un mismo ámbito de conocimiento.

Una vez que los proyectos de normativa legal enunciados en el presente documento hayan sido remitidos por el Gobierno, para su informe, al Consejo de Coordinación Universitaria, procedería adoptar la decisión de paralizar la tramitación de autorizaciones de homologación y de autorización de implantación de nuevas enseñanzas ajustadas a la normativa vigente.

Respecto a la las consecuencias que la implantación de esta reforma del sistema universitario español pueda comportar para los titulados por el sistema actual cabe establecer las siguientes previsiones que se someten a consideración del Consejo de Coordinación Universitaria:

1a ) Con carácter general, los Diplomados, Ingenieros Técnicos, Arquitectos Técnicos, Licenciados, Ingenieros o Arquitectos titulados por el sistema vigente mantendrán todos los derechos, académicos y profesionales, que confieren sus títulos oficiales con carácter indefinido.

$2^{\mathrm{a}}$ ) La transformación de los actuales Diplomados, Ingenieros Técnicos y Arquitectos Técnicos en Licenciados, Ingenieros o Arquitectos del esquema del Espacio Europeo de Educación Superior no será automática y, en cualquier caso, requerirá la homologación previa de las nuevas titulaciones. El Gobierno, previo informe del Consejo de Coordinación Universitaria, establecerá un sistema de pasarelas o formación adicional para facilitar la conversión.

Los requisitos de acceso de estos Diplomados, Ingenieros Técnicos y Arquitectos Técnicos a los estudios de Postgrado que, en sus ámbitos de conocimiento, se diseñen a partir de la implantación del nuevo sistema, pueden ser establecidos bajo una de las dos fórmulas siguientes:

a) exigir que estos titulados únicamente puedan acceder a estos estudios de Postgrado (Master y Doctorado) tras haber obtenido el correspondiente titulo de Grado de Licenciado, Ingeniero o Arquitecto.

b) abrir la posibilidad, además de la anterior, de que, según los estudios de postgrado a los que opten y según hayan quedado definidos en el nuevo sistema los contenidos y créditos de los títulos de Grado correspondientes a su actual titulación, puedan acceder al nivel de Postgrado si cumplen los requisitos de formación adicionales que el Gobierno establezca, a propuesta del Consejo de Coordinación Universitaria, en función de la formación académica previa acreditada y de los contenidos del programa de postgrado. 
3a) Tampoco podrán convalidarse, de forma automática, los actuales títulos de Licenciado, Ingeniero o Arquitecto por los nuevos títulos oficiales de Master. Pero el Gobierno previo informe del Consejo de Coordinación Universitaria podrá aprobar los sistemas de acceso y reconocimiento de estudios de estos titulados en las nuevas enseñanzas de postgrado.

$4^{\text {a }}$ ) Los actuales alumnos de Doctorado podrán continuar sus estudios de tercer ciclo por el sistema en que los hayan iniciado, salvo en lo relativo a tribunales y defensa de la tesis cuya regulación podrá ser modificada por el Gobierno previo informe del Consejo de Coordinación Universitaria.

A medida que vayan implantándose las nuevas enseñanzas de Postgrado, los alumnos que en ese momento se encuentren cursando los estudios de doctorado por el sistema actual y no hayan obtenido el Diploma de Estudios Avanzados podrán continuar sus estudios de Doctorado accediendo a los nuevos programas y solicitando el reconocimiento de los estudios ya realizados.

\section{EPÍLOGO}

En un proceso de tanta transcendencia para el sistema universitario español resulta decisivo no sólo compartir los objetivos finales, sino también aunar los esfuerzos de todos los responsables educativos para su consecución. El reto que comporta su integración en el Espacio Europeo de Educación Superior en modo alguno debe limitarse a meros cambios normativos pues el objetivo al que estos se encaminan no es otro que aprovechar el impulso de esa integración para reforzar los niveles de calidad y competitividad internacional de nuestras enseñanzas universitarias adecuándolas eficazmente a las exigencias de la formación superior de la sociedad contemporánea. Los beneficiarios más directos de las reformas que se proponen serán los propios estudiantes para quienes comportarán ventajas significativas en su formación, movilidad e integración laboral.

En fin, el espacio educativo europeo es un objetivo que debe ser construido entre todos los estados y en el que las universidades españolas deben desempeñar un papel activo. Una construcción que no se orienta a diseñar un perfil homogéneo, sino a armonizar los sistemas educativos respetando la diversidad de culturas nacionales y la autonomía universitaria. 


\section{ANEXO I: DECLARACIÓN DE BOLONIA}

19 de junio de 1999

El proceso europeo, gracias a los logros extraordinarios de los últimos años, se ha convertido en una realidad cada vez más concreta y determinante en la vida de la Unión y de sus ciudadanos. Las perspectivas de ampliación y de intensificación de las relaciones con otros países europeos proporcionan a esa realidad unas dimensiones aún más amplias. Al mismo tiempo, estamos viendo una creciente concienciación, en muchas partes del mundo político-académico y en la opinión pública, de la necesidad de conferir a la construcción europea una articulación diferenciada y completa, reforzando sobre todo sus dimensiones intelectuales, culturales, sociales, científicas y tecnológicas.

La Europa de los conocimientos está ampliamente reconocida como factor insustituible para el crecimiento social y humano, y como elemento indispensable para consolidar y enriquecer la ciudadanía europea, confiriendo a sus ciudadanos las competencias necesarias para afrontar los retos del nuevo milenio, junto con la concienciación de los valores compartidos y de la pertenencia a un espacio social y cultural común.

Basándose en estos planteamientos, la declaración de La Sorbona del 25 de mayo de 1998, subrayó el papel central de las universidades en el desarrollo de la dimensión cultural europea. Asimismo, hizo hincapié en la construcción del espacio europeo de enseñanza superior como instrumento clave en la promoción de la movilidad de los ciudadanos, su ocupabilidad, y el desarrollo global del continente.

Varios países europeos han aceptado la invitación de comprometerse a conseguir los objetivos expuestos en la declaración, tanto los que la firmaron como los que expresaron su acuerdo. La dirección tomada por varias reformas de la enseñanza superior, lanzadas mientras tanto en Europa, es una demostración palpable del compromiso de muchos gobiernos de operar en este sentido.

Por su parte, las instrucciones europeas de enseñanza superior han aceptado el reto y han asumido un papel principal en la construcción del espacio europeo de enseñanza superior, dentro del marco de los principios fundamentales expresados en la Magna Charta Universitatum de Bolonia de 1988. Este hecho es de vital importancia ya que la independencia y autonomía de las universidades garantizan la constante adecuación del sistema de enseñanza superior y de investigación a las necesidades y exigencias de la sociedad y del conocimiento científico.

El rumbo ya está establecido con firmes propósitos. Sin embargo, la consecución de una mayor compatibilidad y comparabilidad de los sistemas de enseñanza superior requiere un impulso constante. Deberíamos apoyarla a través de medidas concretas para realizar avances tangibles. El convenio del 18 de junio, en el cual participaron expertos y estudiosos de todos nuestros países, ha proporcionado indicaciones utilísimas en cuanto a la iniciativa a emprender.

Deberíamos analizar sobre todo el objetivo de incrementar la competitividad internacional del sistema europeo de enseñanza superior. La vitalidad y eficacia de cualquier civilización pueden medirse a través de la atracción que ejerce su cultura sobre otros países. Debemos asegurarnos de que el sistema europeo de enseñanza superior adquiera un grado de atracción que corresponda a nuestras extraordinarias tradiciones culturales y científicas. 
Además de consolidar nuestro apoyo a los principios generales expuestos en la declaración de La Sorbona, nos comprometemos a coordinar nuestras políticas para alcanzar a corto plazo, y en cualquier caso antes del final de la primera década del tercer milenio, los siguientes objetivos, los cuales consideramos de máxima relevancia para el establecimiento del espacio europeo de enseñanza superior y para la promoción mundial del sistema europeo de enseñanza superior:

- La adopción de un sistema de títulos de sencilla legibilidad y comparabilidad, a través de la introducción del Diploma Supplement, con tal de favorecer la employability (ocupabilidad) de los ciudadanos europeos y la competitividad internacional del sistema europeo de enseñanza superior.

- La adopción de un sistema basado esencialmente en dos ciclos principales, respectivamente de primer y segundo nivel. El acceso al segundo ciclo precisa de la conclusión satisfactoria de los estudios de primer ciclo, que duran un mínimo de tres años. El título otorgado al final del primer ciclo será utilizable como cualificación en el mercado laboral europeo. El segundo ciclo debe conducir a un título de master o doctorado como en muchos países europeos.

- El establecimiento de un sistema de créditos - como el modelo ECTS- como medio de promover la movilidad de estudiantes. Los créditos también pueden adquirirse en otros contextos, como la formación permanente, siempre que estén reconocidos por las universidades receptoras en cuestión.

- La promoción de la movilidad; mediante la eliminación de los obstáculos para el pleno ejercicio de la libre circulación con especial atención a lo siguiente:

- Para los estudiantes: el acceso a oportunidades de estudio y formación, y a servicios relacionados.

- Para profesores, investigadores y personal técnico-administrativo: el reconocimiento y valorización de períodos de investigación en contextos europeos relacionados con la docencia y la formación, sin perjuicio para los derechos adquiridos.

- La promoción de una colaboración europea en la garantía de calidad con vistas al diseño de criterios y metodologías comparable.

- La promoción de las dimensiones europeas necesarias en la enseñanza superior, sobre todo en lo que respecta al desarrollo curricular, colaboración interinstitucional, planes de movilidad y programas integrados de estudio, formación e investigación.

Nosotros nos comprometemos a alcanzar estos objetivos - dentro del marco de nuestras competencias institucionales y con pleno respeto a la diversidad de culturas, lenguas, sistemas nacionales de enseñanza y autonomía universitariapara consolidad el espacio europeo de enseñanza superior. Con este fin, perseguiremos las vías de colaboración intergubernamental y la de organismos europeos no gubernamentales con competencias en asuntos de enseñanza superior. Esperamos que las universidades contesten pronto y de manera positiva, y que contribuyan activamente al éxito de nuestra iniciativa.

Convencidos de que el establecimiento del espacio europeo de enseñanza superior requiere el apoyo, vigilancia y adaptación constante a nuestras necesidades en plena evolución, decidimos volver a reunirnos dentro de dos años para evaluar los avances conseguidos y las nuevas medidas a tomar. 
ANEXO II: LEY ORGÁNICA DE UNIVERSIDADES

TÍTULOXIII

ESPACIO EUROPEO DE ENSEÑANZA SUPERIOR

\section{Artículo 87. DE LA INTEGRACIÓN EN EL ESPACIO EUROPEO DE ENSEÑANZA SUPERIOR}

En el ámbito de sus respectivas competencias el Gobierno, las Comunidades Autónomas y las Universidades adoptarán las medidas necesarias para la plena integración del sistema español en el espacio europeo de enseñanza superior.

\section{Artículo 88 DE LAS ENSEÑANZAS Y TÍTULOS}

1. A fin de promover la más amplia movilidad de estudiantes y titulados españoles en el espacio europeo de enseñanza superior, el Gobierno, previo informe del Consejo de Coordinación Universitaria, adoptará las medidas que aseguren que los títulos oficiales expedidos por las Universidades españolas se acompañen de aquellos elementos de información que garanticen la transparencia acerca del nivel y contenidos de las enseñanzas certificadas por dicho título.

2. No obstante lo dispuesto en el artículo 37, y con el fin de cumplir las líneas generales que emanen del espacio europeo de enseñanza superior, el Gobierno, previo informe del Consejo de Coordinación Universitaria, establecerá, reformará o adaptará las modalidades cíclicas de cada enseñanza y los títulos de carácter oficial y validez en todo el territorio nacional correspondiente a las mismas.

Cuando estos títulos sustituyan a los indicados en el citado artículo 37, el Gobierno, previo informe del Consejo de Coordinación Universitaria, determinará las condiciones para la homologación de éstos a los nuevos títulos, así como para la convalidación o adaptación de las enseñanzas que los mismos refrenden.

3. Asimismo, el Gobierno, previo informe del Consejo de Coordinación Universitaria, establecerá las normas necesarias para que la unidad de medida del haber académico, correspondiente a la superación de cada una de las materias que integran los planes de estudio de las diversas enseñanzas conducentes a la obtención de títulos de carácter oficial y validez en todo el territorio nacional, sea el crédito europeo o cualquier otra unidad que se adopte en el espacio europeo de enseñanza superior, y para que las Universidades acompañen a los títulos oficiales que expidan, en desarrollo de lo dispuesto en el artículo 34 de la presente Ley, el suplemento europeo al título.

4. El Estado, las Comunidades Autónomas y las Universidades fomentarán la movilidad de los estudiantes en el espacio europeo de enseñanza superior a través de programas de becas y ayudas y créditos al estudio o, en su caso, complementando los programas de becas y ayudas de la Unión Europea.

\section{Artículo 89 \\ DEL PROFESORADO}

1. El profesorado de las Universidades de los Estados miembros de la Unión Europea que haya alcanzado en aquéllas una posición equivalente a las de Catedrático o Profesor Titular de Universidad o de Catedrático o Profesor Titular de Escuelas Universitarias 
será considerado habilitado a los efectos previstos en esta Ley, según el procedimiento y condiciones que se establezcan reglamentariamente por el Gobierno, previo informe del Consejo de Coordinación Universitaria.

2. El profesorado al que se refiere el apartado 1 podrá formar parte de las Comisiones a que se refiere el artículo 57 de la presente Ley y, si las Universidades así lo establecen en sus Estatutos, de las Comisiones encargadas de resolver los concursos para el acceso a los cuerpos docentes universitarios.

3. A los efectos de la concurrencia a las pruebas de habilitación . y concursos de acceso a los cuerpos de funcionarios docentes universitarios y a las convocatorias de contratos de profesorado que prevé esta Ley, los nacionales de Estados miembros de la Unión Europea gozarán de idéntico tratamiento, y con los mismos efectos, al de los nacionales españoles.

Lo establecido en el párrafo anterior será de aplicación a los nacionales de aquellos Estados a los que, en virtud de Tratados Internacionales celebrados por la Unión Europea y ratificados por España, sea de aplicación la libre circulación de trabajadores en los términos en que ésta se encuentra definida en el Tratado Constitutivo de la Comunidad Europea.

4. El Estado, las Comunidades Autónomas y las Universidades fomentarán la movilidad de los profesores en el espacio europeo de enseñanza superior a través de programas y convenios específicos y de los programas de la Unión Europea. 


\section{ÍNDICE}

1. INTRODUCCIÓN

2. LOS NUEVOS RETOS DEL SISTEMA UNIVERSITARIO ESPAÑOL

3. EL ESPACIO EUROPEO DE EDUCACIÓN SUPERIOR

4. LA LOU Y EL ESPACIO EUROPEO DE EDUCACIÓN SUPERIOR

5. PROPUESTAS PARA LA INTEGRACIÓN DEL SISTEMA UNIVERSITARIO ESPAÑOL EN EL ESPACIO EUROPEO DE EDUCACIÓN SUPERIOR

5.1. La implantación del sistema de créditos europeos

5.2. La adaptación de las enseñanzas y títulos oficiales

5.2.1. El primer nivel: el Grado

5.2.2. El segundo nivel: el Postgrado

5.3. El suplemento europeo al título: un elemento de transparencia

5.4. Acreditación académica y calidad

6. LA CORRESPONSABILIDAD INSTITUCIONAL EN LA PUESTA EN MARCHA DEL PROCESO

7. LA TRANSICIÓN DE LAS TITULACIONES ACTUALES AL NUEVO SISTEMA DE LAS ENSEÑANZAS UNIVERSITARIAS

8. EPÍLOGO

\section{ANEXOS}

- Declaración de Bolonia

- Título XIII de la Ley Orgánica de Universidades 